\title{
Neuro Invasive Viruses the Silent and the Slow Killer
}

\author{
${ }^{1}$ Prof. Dr. Shahid Hussain Sheikh, ${ }^{2}$ Saima Chaudhary M.S., \\ ${ }^{I}$ MBBS, Ph.D., ${ }^{2}$ M.Phil Clinical Psychology. \\ ${ }^{1,2}$ Dept. of Neurology/Neuro Virology United Christian Hospital, Gulberg III, Lahore.
}

In 1980's the average life expectancy was 60 years, whereas, now it is less than 46 years. Clinically, common ailments are being presented comparatively 10 to 15 years early than 50 years ago.

At the outset, general healthcare is becoming a growing challenge everywhere on the planet. Most every country is recording high numbers in the world's leading causes of deaths. Emerging viral breakouts and other infectious diseases are also posing serious threats and baffling the research scientists worldwide.

As we all know that every system of our body consists of multiple organs and tissues and membranes and cells that all are managed and controlled by our brain for a normal function. The ailments presented are taken into an account as a unique local problem understanding the "signs \& Symptoms", the prescription is made to rectify the challenge. Most of the times the grass root of the problem (Etiology) is unknown. Symptomatic treatment buys the time for the immunity to kick start the body's own defense system to rectify the ailment.

Many patients with fair immunity do benefit from this practice, those who chronically dealing with long term ailments i.e. Hypertension, Cholesterol, Stroke, G,I, Ulcer and many more, are simply hooked on the disease pacifying drugs. What to say about the genetic disorders, developmental disorders, unexplainable physical challenges and condition of infants, children and adults alike.

Clearly, the time has come now to realize that, there is an urgent and tremendous need to go back to the drawing table and expand our understanding of the disease process. At this time, we are relying on "Signs \& Symptoms" and may be treat the disease to the system or organ or tissue or the cellular level.

We are left with no choice but to cross the cell and go to the "Nucleus Level" and understand the DNA \& RNA pathology. Specially, those cells that perform the management \& control of the whole system.

A new specialty called "Neuro Virology" reveals that continuous Viral RNA proliferation in given neurons ultimately reduce the controlling capability of those neurons and begins to give rise to the symptoms of the ailing system; while the immunity also becomes compromised.

This phenomenon starts the multi functional and structural disorders (The Sheikh's Syndrome).

A group of scientists of University of California at Irvine first documented with the aid of Atomic Force Microscopy (AFM), the real time cellular infection with the lesion and the virion at site. This establishes the existence of the primary disease. While treating any secondary disease, one must not ignore the primary root cause at the controlling neuron level.

Local organ and tissue level insult and presented symptomatology always reflects the malfunction of their controlling neurons. Physically inflicted injury of course is always another issue and is dealt with accordingly i.e cuts, burns or bruises.

Currently, many patients inflicted with the neuro invasive viruses are seen in the clinics everyday presenting transient and permanent multiple physical and psychological challenges.

Since the conventional treatments do not address the continuous viral RNA proliferation, therefore, the sub-nucleus neuro pathology continues to grow at the cost of defying immunity; this eventually results in morbidity and mortality.

A 10 year old boy happy go lucky, living a common life as any other boy of same age, interests, physical activities and social demeanor. All of a sudden starts presenting the following symptoms.

- Unexplainable tiredness, fatigue

- Frequent daily episodes of Irritability without any reason

- Transient pain in multiple areas of body

- Watery eyes

- Isolation, Solitaire

- Avoiding to talk

- Frequent Monoclonic Fits

Parents commonly ignore these early challenges. But, the vigilant parents of the patient took him to the local physician, where he was given some pain killers and sent home. Few weeks go by and these symptoms persisted along with the following challenges.

- Now experiencing difficulty in Studies 
- Difficulty in normal walk

- Difficulty in speech, cries most of the time

- Difficulty in living a normal life

At this stage, parent's quest for right diagnosis and treatment expanded to multiple pediatricians, internists, orthopedics and leading Psychiatrists, Psychologists and neurologist from Quetta to Karachi, Islamabad and finally Lahore. No one could pin point the root cause and the treatment for it.

Parents resorted to the faith healers, naturopaths, homeopaths. One after the other, town to town but no relief. The boy now in diapers, 4 years passed, resources exhausted.

The Boy is now in the following conditions.

- Can Not Walk

- Can not speak

- General Epileptic Episodes

- Lost a lot of weight

- $\quad$ Feet twisted, Hands twisted

- Blistered Eyes

- Difficulty in Breathing

- Serious Anemia

- Memory Lapses

- Minimal Physical Response

Leading neurologist have carried out CT scan and MRI and made the following diagnosis.

- Cerebral Atrophy

- Cerebral Palsy

- Diversion

- Odd Behavior

- Anemia

- Diffused Cortical Damage

- Encephalitis

- Borderline Status Epileptica

No treatment, other than blood transfusion to keep the patient alive.

Parents hear something about NeuroVirology and carry the 15 year old boy to the Department of Neurology at the United Christian Hospital, Lahore and meet with the Prof. Dr. Shahid Hussain Sheikh, the country's leading Neuro Virologist to examine the boy. Prof. Sheikh examines the patient and interview the parent's and runs the Neuro Virology Blood Tests and finds the following.

- Cytomegaloviral (CMV) RNA induced Multifocal Sub-Nucleus Neuronalitis, affecting Multiple systems and organs including:

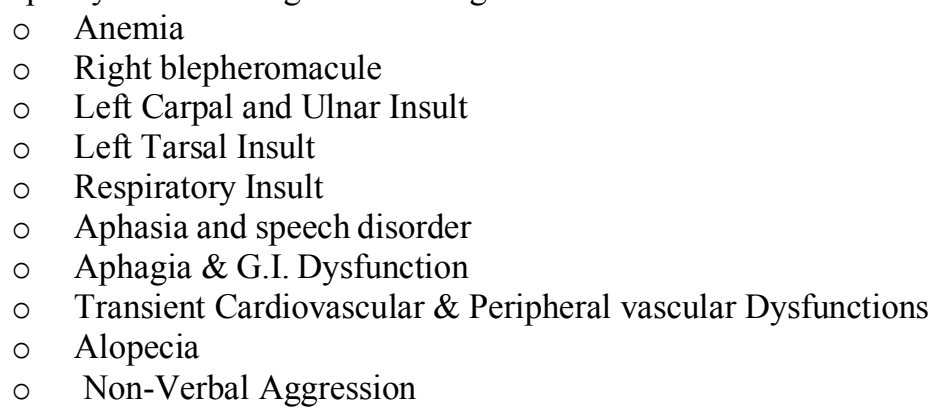

The boy was admitted for the treatment of the above neuro viral pathology. After 3 days of intensive antiviral therapy the patient started responding physically and anemia started resolving.

Furthermore, on the fifth day, the patient started speaking 3 words sentences with his mother and started facial and hands movements. It's like frozen nerves begin to thaw.

On the $7^{\text {th }}$ day complete speech came back. Now the patient directly was able to communicate about his ailments, which help us further fine tune our treatment for the neuro invasive viral pathology. 
In the parent's interview, it was found that, the mother was infected with the HSV right before her pregnancy. It was linked that the controlling neurons which are responsible to secret the neuro secretory enzymes which stimulate the Pituitary gland, would have carried out some influence of the Neuro invasive viral RNA's from their invaded nucleus.

Therefore, the molecular integrity of the stimulating enzymes must have been compromised.

In turn, stimulating hormones from the pituitary also carried the grave influence of the viral RNA's down to the ovaries and testes for the production of the sperm and the ovum.

Neuro Viral RNA's had to make its journey through the chromosomal DNA / RNA's, either from mother or father side. In this case, due to the mothers state of HSV infection just prior topregnancy warranted this invasion.

Because the environment is now very favorable for the proliferation of the zygote, neuro invasive viral RNA's also are designed to continuously transcribe. Though it will never become a part of any developing structure of the fetal tissues or membrane or cells, but will occupy the intracellular space/s in the developing tissues \& Membranes of different organs, specially Brain and spinal cord.

If the pregnancy surpassed against its grave influence and did not result into miscarriage, then delivery might become challenging, if passed that successfully, then early childhood challenges come about. If that surpasses, then, as soon as the child's own immunity slows down, most all of the above conditions begin to align and cause the child's life very challenging.

Children experience and demonstrate a variety of physiological and behavioral issues that

Conventional allopathy labels them as congenial/ developmental or genetic disorders and shelf them to further suffer with it.

As a matter of fact, majority of the world's unsuccessful pregnancies and deliveries go through with this neuro viral tragedy at one point in time during 9 months, leaving emotional scars on the mothers.

Many "successful deliveries", later demonstrate the under achieving, low I.Q., aggressive behavior, School drop out children and adults due to the Neuro invasive viral infliction some point in time in their life.

The adults who have been known to have carried this

pathology, do have a variety of physical, psychological and civil/ social challenges in their lives, including but not limited to the domestic violence, aggressors, hyperactive, schizophrenic, personality disorders, bipolar disorder etc.

The good news is that the new research as "Sheikh's Syndrome" shows some hope to help control what is already done, clean up before planning to make a baby (Specially who had multiple sex partners) and monitor the pregnancy with the help of a neuro virologist.

This case study evidences the grass root problem that started the soft symptoms 4 to 5 years before it became a full physical, psychological and anatomical challenge. Indeed, it is the silent and the slow killer. 